\title{
Idiopathic Pulmonary Vein Thrombosis in a Patient with Chronic Obstructive Pulmonary Disease
}

\author{
James Allan Douglas Shand, Kate Siobhan Bradley, Cameron Kinjun Manfred Willy Schauer
}

Department of Medicine, Whangarei Hospital, Whangarei, New Zealand

\begin{abstract}
We present the case of a 67 -year-old man who presented to our hospital with acute shortness of breath. He was initially treated for a presumed infective exacerbation of chronic obstructive pulmonary disease (COPD) but did not respond to standard therapy. Further investigations were performed and revealed a large pulmonary vein thrombus. Clinical, laboratory, and radiological investigation did not identify an underlying cause for his thrombus. Idiopathic pulmonary vein thrombosis is much rarer than the more well-known pulmonary embolism and is worthy of consideration as an uncommon cause of pulmonary decline. To our knowledge, this is the first case report in the English literature of idiopathic pulmonary vein thrombus occurring in the setting of known COPD.

Keywords: Chronic obstructive pulmonary disease, idiopathic pulmonary vein thrombosis, pulmonary vein thrombosis
\end{abstract}

\section{INTRODUCTION}

We present a case of pulmonary vein thrombosis (PVT) occurring in a patient with chronic obstructive pulmonary disease (COPD). In contrast to the more well-known pulmonary embolism, idiopathic pulmonary vein thrombosis is an exceedingly rare condition with only a handful of case reports in the English literature. This is the first time that PVT has been diagnosed in a patient with COPD, and we believe the diagnostic journey serves as an interesting reminder to the reader about the importance of a broad differential diagnosis.

\section{CASE PRESENTATION}

A 67-year-old man presented with acute shortness of breath and decreased exercise tolerance. He was an ex-30-pack-year smoker whose past medical history included chronic obstructive pulmonary disease, hypertension, and a hemi-colectomy conducted 25 years earlier for a bowel abscess. Upon arrival at our hospital, he was hypoxic $\left(\mathrm{SpO}_{2}, 83 \%\right)$, breathing ambient air; tachypnoeic (respiratory rate, 30 breaths/min) and tachycardic with a heart rate of 117 beats $/ \mathrm{min}$. His blood pressure was $147 / 107 \mathrm{mmHg}$. Auscultation of the lungs revealed coarse crackles to the midzones bilaterally. The remainder of the cardiorespiratory examination was unremarkable. A chest radiograph showed hyperinflated lungs but no acute infective changes. Relevant blood component levels on arrival were as follows: C-reactive protein $44 \mathrm{mg} / \mathrm{L}(0-5)$, neutrophil count $4 \cdot 1 \times 10^{9} / \mathrm{L}\left(4-11 \times 10^{9}\right)$, and NT-ProBNP $41 \mathrm{pmol} / \mathrm{L}(<35)$. The patient was treated for a presumed infective exacerbation of COPD with IV ceftazidime based on sensitivities from his previous sputum culture results.

He did not improve clinically with antibiotic therapy so further investigations were undertaken. Transthoracic echocardiography was unremarkable aside from mild right ventricular enlargement. Further blood tests revealed a D dimer of $2170 \mathrm{mcg} / \mathrm{L}(0-700)$; therefore, computed tomography pulmonary angiogram (CTPA) was performed. This revealed emphysema, widespread bronchiectatic changes, and a 44-mm thrombus occluding the left inferior pulmonary vein (Figure 1). The patient had no known prothrombotic risk factors. An extended CT scan of the abdomen and pelvis revealed no occult malignancy. Serum procoagulant screening was performed and showed no abnormalities in the pro- 


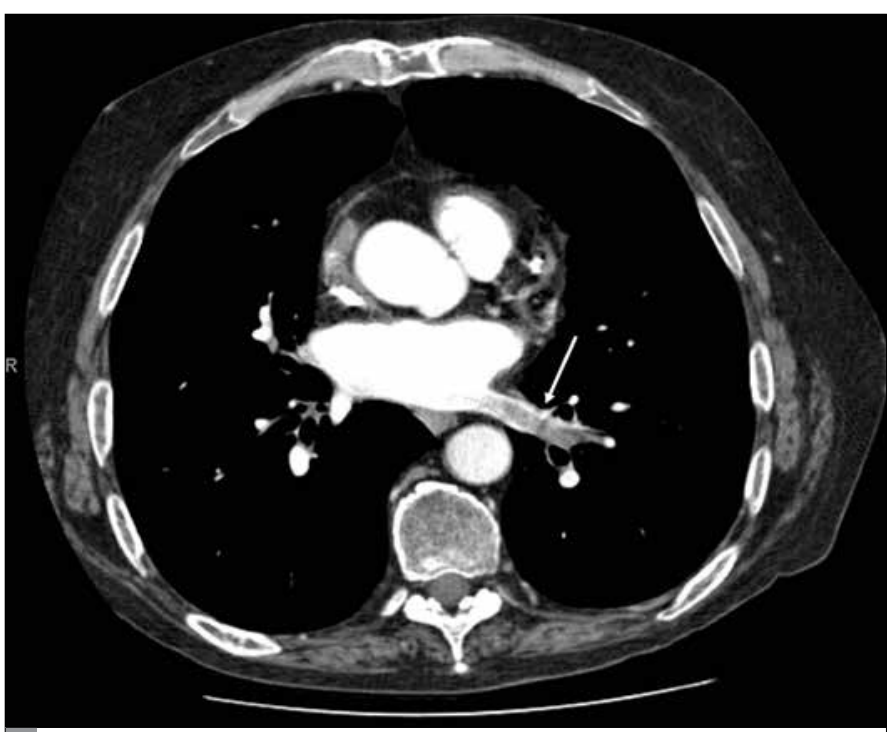

Figure 1. Computed tomography pulmonary angiogram revealing a 44-mm filling defect in the left inferior pulmonary vein (solid arrow)

thrombin ratio, activated partial thromboplastin time, Russel's viper venom time, Kaolin clotting time, and functional Protein $\mathrm{C}$ and Protein S levels. A factor $V$ Leiden mutation was not detected, and there was no abnormal activated protein $C$ resistance. Idiopathic pulmonary vein thrombosis was diagnosed. Antibiotics were discontinued, and the patient was started on a long course of oral anticoagulation and discharged.

\section{DISCUSSION}

Pulmonary vein thrombosis is a rare condition, infrequently described in the literature. It is defined by the formation of a blood clot in situ within the pulmonary vein, resulting in blood flow disruption. It is potentially life-threatening and requires a high index of suspicion for diagnosis.

Acquired PVT is usually associated with atrial trauma and occurs most commonly following lung transplant, lobectomy (1), and pulmonary vein isolation procedures (2). Other known causes are pulmonary neoplasm (more commonly, primary lung cancer), fibrosing mediastinitis, and mitral stenosis with a left atrial clot (3). Idiopathic PVT is very rare, and its presence in the literature is limited to a small number of case reports.

The mechanism underlying PVT is poorly understood, but it is thought that iatrogenic or immunologic damage to the vein wall may stimulate thrombosis formation (1). In patients with malignancy, a hypercoagulable state or mechanical compression of the pulmonary vein may contribute to thrombosis (4).

Chronic obstructive pulmonary disease is a known moderate risk factor for the development of thrombus in the form of deep vein thrombosis and pulmonary embolism (5). The prevalence of venous thromboembolism (VTE) during COPD exacerbations is reported to range from $5 \%$ to $29 \%$ (6). A number of factors, including a systemic inflammatory state, are thought to contribute to this increased risk (7). Despite the association between COPD and pulmonary arterial thrombus, we were unable to find any documented cases of PVT in patients with COPD. We do, however, postulate that there may be an as-yet unknown causal link between the procoagulant chronic inflammatory state of COPD and the development of PVT.

The signs and symptoms of PVT are non-specific, making it a difficult clinical diagnosis. It can present acutely with cough, dyspnoea, pleuritic chest pain, and haemoptysis or as a progressive deterioration in respiratory function $(1,8)$.

Diagnosis may be established with transesophageal echocardiography (TEE), $\mathrm{CT}$, or magnetic resonance imaging (MRI). A thrombus reaching the large distal veins and left atrium may be seen on TEE, but the diagnosis requires a high level of skill. CT is a non-invasive and reproducible diagnostic tool and is the recommended imaging modality for PVT (1). MRI plays a role in distinguishing tumor from thrombus in select cases (9).

No treatment protocols have been generated for the management of idiopathic PVT. However, successful clot resolution has been reported with anticoagulation $(1,2)$. Early diagnosis and treatment can help to prevent the other potential complications, including pulmonary gangrene, peripheral embolus, and massive haemoptysis (3).

In summary, pulmonary vein thrombus is a rare condition but is worthy of consideration as an unusual cause of both progressive pulmonary deconditioning and acute respiratory decline. To our knowledge, this case report represents the first documentation of PVT occurring in the context of COPD, although the true prevalence of this disease remains unknown. We feel that this case can serve as a reminder to physicians of the importance of a broad differential diagnosis when assessing and managing patients, particularly those whose clinical course differs from that expected.

\section{CONCLUSION}

We present the first published case report of PVT occurring in a patient with known COPD. This is a rare condition, and our case report serves as a reminder of the importance of a broad differential diagnosis required when assessing patients.

Informed Consent: Written informed consent was obtained from the patient who participated in this case.

Peer-review: Externally peer-reviewed.

Author Contributions: Concept - J.A.D.S., K.S.B., C.K.M.W.S.; Design - T.Ç., I.S.K.; Literature Search - J.A.D.S., K.S.B., C.K.M.W.S.; Writing Manuscript - J.A.D.S., K.S.B., C.K.M.W.S.; Critical Review - J.A.D.S., K.S.B., C.K.M.W.S.

Conflict of Interest: No conflict of interest was declared by the authors.

Financial Disclosure: The authors declared that this study has received no financial support.

\section{REFERENCES}

1. Schulman LL, Anandarangam T, Leibowitz DW, Ditullio MR, McGregor CC, Galantowicz ME, et al. Four-year prospective study of pulmonary venous thrombosis after lung transplantation. J Am Soc Echocardiogr 2001; 14: 806-12. [CrossRef]

2. Hovaguimian H, Morris JF, Gately HI, Floten HS. Pulmonary vein thrombosis following bilobecomy. Chest 1991; 99: 1515-6. [CrossRef]

3. Cavaco RA, Kaul S, Chapman T, Casaretti R, Philips B, Rhodes A, et al. Idiopathic pulmonary fibrosis associated with pulmonary vein thrombosis: a case report. Cases J 2009; 2: 9156. [CrossRef]

4. Kim NH, Roldan CA, Shively BK. Pulmonary vein thrombosis. Chest 1993; 104: 624-6. [CrossRef] 
5. Torbicki A, Perrier A, Konstantinides S, Agenlli G, Galie N, Pruszczyk P, et al. Guidelines on the diagnosis and management of acute pulmonary embolism: the Task Force for the Diagnosis and Management of Acute Pulmonary Embolism of the European Society of Cardiology (ESC). Eur Heart J 2008; 29: 2276-315. [CrossRef]

6. Kim V, Goel N, Gangar J, Zhao H, Ciccolella DE, Silverman EK, et al. Risk factors for venous thromboembolism in chronic obstructive pulmonary disease. Chronic Obstr Pulm Dis (Miami) 2014; 1: 239-49. [CrossRef]
7. Ambrosetti M, Ageno W, Spanevello A, Salerno M, Pedretti RF. Prevalence and prevention of venous thromboembolism in patients with acute exacerbations of COPD. Thromb Res 2003; 112: 203-7. [CrossRef]

8. Wu JP, Wu Q, Yang Y, Du ZZ, Sun HF. Idiopathic pulmonary vein thrombosis extending to left atrium: a case report with a literature review. Chin Med J (Engl) 2012; 125: 1197-200.

9. Selvidge SD, Gavant ML. Idiopathic pulmonary vein thrombosis: detection by CT and MR imaging. Am J Roentgenol 1999; 172: 1639-41. [CrossRef] 